\title{
Current status on robotic assisted myomectomy
}

\author{
Imrich Kiss, Pavla Svobodova, Lubos Karasek, Bohuslav Svoboda \\ Department of Gynecology, 3rd Faculty of Medicine Charles University and Military University Hospital, Prague 16902, Czech \\ Republic.
}

Correspondence to: Dr. Imrich Kiss, Department of Gynecology 3rd Faculty of Medicine, Charles University, Military University Hospital, U Vojenske nemocnice 1200, 16902 Praha 6, Prague 16902, Czech Republic. E-mail: kiss.imrich@gmail.com

How to cite this article: Kiss I, Svobodova P, Karasek L, Svoboda B. Current status on robotic assisted myomectomy. Miniinvasive Surg 2021;5:55. https://dx.doi.org/10.20517/2574-1225.2021.70

Received: 25 May 2021 First Decision: 8 Oct 2021 Revised: 7 Nov 2021 Accepted: 22 Nov 2021 Published: 8 Dec 2021

Academic Editors: Giulio Belli, Simone Ferrero Copy Editor: Xi-Jun Chen Production Editor: Xi-Jun Chen

\begin{abstract}
Uterine leiomyomas are common benign solid tumors of the uterus. While the presence of fibroids is rarely life threatening, they are associated with symptoms affecting quality of life and fertility. Myomectomy is a standard fertility-sparing surgery which should be considered for women suffering from fibroid-related symptoms who do not desire hysterectomy or any alternative treatment option. While open surgery is thought to be reserved for large and numerous myomas, mini-invasive methods as laparoscopy and robot-assisted surgery have evolved in the hands of experienced surgeons to also deal with these more complex cases. Robotic myomectomy has its advantages in lower blood loss, fewer complications, and shorter hospital stay over open surgery, whereas the comparison outcomes with laparoscopic myomectomy are still uncertain. Advantages of the wristed instruments, three-dimensional vision along with the incorporation of correct surgical techniques could emphasize the benefits of the robotic assisted approach in large and numerous myoma cases. Careful and detailed assessment should precede the surgery to recognize risks and steps to reduce operation time, which tends to be the most presented drawback of robotic myomectomy. As the tendency of robot-assisted surgeries is growing, many authors share their experience or publish comparison studies with other surgical methods. Our article describes the current status concerning robotic myomectomy, reviewing publications from the past five years (2016-2021).
\end{abstract}

Keywords: Robotic surgery, robotic myomectomy, robot-assisted myomectomy, surgical techniques

\section{INTRODUCTION}

Uterine leiomyomas (uterine fibroids) are a common benign smooth muscle tumors of the uterus that

(C) The Author(s) 2021. Open Access This article is licensed under a Creative Commons Attribution 4.0 International License (https://creativecommons.org/licenses/by/4.0/), which permits unrestricted use, sharing adaptation, distribution and reproduction in any medium or format, for any purpose, even commercially, as long as you give appropriate credit to the original author(s) and the source, provide a link to the Creative Commons license, and indicate if changes were made. 
affects up to $70 \%$ of women until reaching menopause ${ }^{[1]}$. Risk factors include ethnicity, parity, early menarche, late menopause, family history, obesity, and hypertension ${ }^{[2]}$. While the presence of myomas are rarely life threatening, they are associated with symptoms affecting quality of life such as abnormal bleeding, pelvic pain, and urinary tract problem $s^{[3]}$. In addition, myomas might also be linked with adverse pregnancy outcomes such as infertility, preterm birth, or postpartum hemorrhage ${ }^{[4]}$. Diagnosis is made based on pelvic bimanual examination, ultrasonography, magnetic resonance imaging, or hysteroscopy. According to the localization of the lesion (submucosal, intramural, or subserous), myomas are further defined by the International Federation of Gynecology and Obstetrics (FIGO) subclassification system to better describe their relation to the uterus and help select the appropriate therapeutical approach alongside other factors such as size, number of lesions, reproductive plans, surgeons' skill, etc. ${ }^{[5]}$. Almost one third of women with myomas seek medical help and request treatment. The current medical strategies offer surgical interventions (myomectomy, hysterectomy, and occlusion of uterine arteries) or their alternatives (uterine artery embolization, high-frequency magnetic resonance-guided focused ultrasound, and ultrasound-guided radiofrequency ablation). Selective progesterone receptor modulators such as ulipristal acetate can be used as a preoperative option or as a pharmacological therapy to reduce symptomatology as an alternative to surgical treatment ${ }^{[0,7]}$.

Myomectomy is a standard fertility sparing surgical method and should be considered for women with fibroid related symptoms who do not desire hysterectomy. While open surgery (laparotomy) is thought to be reserved for large and numerous myomas, mini-invasive methods as laparoscopy and robot-assisted surgery have evolved in the hands of experienced surgeons to also deal with these more complex cases. As the tendency of robot-assisted surgeries is growing, many authors share their experience or comparisons with other surgical methods. In our center, we specialize on all surgical modalities, although in the majority of cases preferring mini-invasive methods. While hysterectomy is still our leading procedure on a robotic system, in the past years, the number of robotic myomectomies is rapidly growing, mostly for complex cases. Our goal when writing this article was to research the current literature to support our shift from laparoscopic to robotic myomectomies. Articles indexed with "robotic myomectomy, robot-assisted myomectomy" and published from 2016 to October 2021 were retrieved from PubMed and reviewed for relevancy. Our article presents the reader up-to-date consolidated information concerning the quickly evolving technique of robotic myomectomy.

\section{COMPARISON STUDIES}

Robotic myomectomy is favorable in less complications, blood loss and hospital stay compared to open surgery, while it is becoming a preferred modality in more complex cases to conventional laparoscopy.

The meta-analysis conducted by Wang et al. ${ }^{[8]}$ including 20 studies (2852 patients) compared robotic, laparoscopic, and open myomectomy. The results show that robotic myomectomy is associated with fewer complications and lower blood loss than the other modalities. It also showed lower conversion rate and less bleeding than laparoscopic myomectomy and lower postoperative pain score than open surgery. In a retrospective study by Ranes et al. ${ }^{[9]}$, longer operation time is stated as the biggest drawback in comparison with open surgery, which could outweigh shorter hospital stay. A mean operative time difference of 84 min (95\%CI: 60.41-109.29) in favor of open myomectomy against robotic assisted was observed in a metaanalysis by Iavazzo et al. ${ }^{[10]}$. On the other hand, robotic assisted myomectomy showed superiority in lower blood loss [92.78 mL/operation (95\%CI: 47.26-138.29)], need for transfusion (981 patients; OR = 0.20; 95\%CI: 0.09-0.43), total complications (1101 patients; OR = 0.31; 95\%CI: 0.11-0.87), and length of hospital stay [1.84 days/patient (95\%CI: 1.40-2.29)]. No significant difference was found in operating time, estimated blood loss, need for transfusion, number of complications, and length of hospital stay between robotic 
assisted and conventional laparoscopic myomectomy.

In a review study considering open or mini-invasive way of entrance in myoma enucleation, comparable outcomes in estimated blood loss, complications, and duration of hospital stay were reached between laparoscopy and robotic surgery ${ }^{[1]}$. The operative time of robotic myomectomy was stated as longer, as in previous studies. Demanding surgical skills for larger myomas and unfavorable localization and higher economic burden were stated as additional limiting factors for robotic myomectomy. The authors cited an older systematic review ${ }^{[12]}$, in which short-term benefits such as blood loss, need of blood transfusion, and hospitalization were significantly lower in robotic assisted myomectomy, while open surgery showed to be preferable in operating time and costs. Gingold et al. ${ }^{[13]}$ reviewed the myoma management in conventional $v s$. robotic assisted myomectomy. The outcomes of the reviewed studies show that robotic surgery was preferred in more complex cases, in which easier maneuverability of the wristed robotic instruments and three-dimensional visualization helped in better dissection, suturing, and application of hemostatic techniques. Nevertheless, these findings tend to be biased by surgeons' experience and inclination to select more difficult cases for robotic surgery and should not be considered as outcome-based evidence to prioritize robotic myomectomy.

When patients were questioned about their symptoms and health quality the morning before and one year after laparoscopic and robotic myomectomy, both groups showed a significant reduction in symptoms and improvement in quality of life without statistical difference between the two methods of surgery ${ }^{[14]}$.

In the last few years, the dominant comparison studies are between multiport $v s$. single-site/single-port robotic myomectomies. The consensus is that multiport myomectomy is preferred for larger myomas, while single-site is feasible for selected patients with less complicated cases, and both methods are associated with low rates of intra- and post-operative complication ${ }^{[15,16]}$. In a very recent review, no significant differences were found in operating time, blood loss, and total complication rate ${ }^{[17]}$.

\section{OPERATING TIME}

Generally, robot-assisted surgeries tend to be longer because of the necessary docking of the robotic arms before the actual surgery begins. For myomectomies, the use of the wristed instruments should speed up the suturing time, which, in comparison with laparoscopy, is a common obstacle even in experienced surgeons. This hypothesis was disproved, when robotic myomectomies showed similar operating time with laparoscopic ones regardless of the number of myomas removed ${ }^{[18]}$. It seems that the difficulty of docking and lack of tactile feedback during enucleation is compensated with easier and faster suturing in robotic myomectomy.

In a very recent study, factors related to the total operative time were body mass index (BMI), number of myomas, total myoma weight, location of dominant myoma, type of da Vinci robotic system (Xi vs. S), intraoperative uterine cavity exposure, blood loss, and total hospitalization period ${ }^{[19]}$. To the contrary, all of the above-mentioned factors, except for the location of dominant myoma and type of robotic system, are also associated with console time. Age, parity, previous surgeries, surgical indication, and size of the dominant myoma were not associated with total operating time. In the analysis of 242 cases, the number of myomas (5-9 vs. $\geq 10$ ) and surgeon's experience were the only two factors that were positively correlated with operation time. Furthermore, the number of myomas and maximal myoma diameter were positively correlated with estimated blood loss ${ }^{[20]}$. 
Movilla et al. ${ }^{[21]}$ proposed a preoperative calculator to predict the total operative time of myomectomies. Factors significantly associated with the length of surgery are age, diabetes mellitus, uterine volume, number of myomas generally and those more than $3 \mathrm{~cm}$, diameter of the dominant myoma, and surgeons' experience. On the other hand, BMI, hypertension, previous surgeries, location, and classification of the myomas do not affect the operative time.

The significantly reduced time of single-site procedure can be acquired by combining the advantages of the laparoscopic enucleation and robotic assisted suturing called hybrid robotic single-site myomectomy ${ }^{[22,23]}$.

The operating time also depends on the experience of the surgeon and the OR team. Robotic myomectomies have a steep learning curve, with the operating time significantly reducing after 10 cases $^{[24]}$.

\section{LARGE/HEAVY/MULTIPLE MYOMAS}

Several case studies show the enucleation of huge myomas (the biggest being $28 \mathrm{~cm}$ and $3.2 \mathrm{~kg}$ ), while pushing the limits of robotic assisted techniques ${ }^{[25,26]}$. These cases confirm the efficiency, reliability, and safety of the robotic approach in well-selected cases regardless of the size of the fibroids. The major advantages of robotic surgery in comparison to abdominal is shorter hospital stay with faster recovery and less blood loss. Wristed instruments enabling a larger range of movements in a limited abdominal space blocked by the enlarged uterus and easier suturing of extensive uterine defects after enucleation are the assets of robotic surgery in contrast with laparoscopy resulting in lower conversion rate. A retrospective study of Lee et al. ${ }^{[27]}$ compared robot-assisted myomectomies (RAM) with abdominal myomectomies (AM) in myomas larger than $10 \mathrm{~cm}$ and heavier than $250 \mathrm{~g}$. While the operating time was significantly longer in RAM than AM (164 min vs. $108 \mathrm{~min}$ ), hospital stays were shorter (2.68 RAM days vs. 4.13 AM days). Shortterm postoperative complications such as fever or bleeding were lower in RAM than AM ( $26 \%$ vs. 54\%). In a retrospective study, outcomes of robotic myomectomies of patients with large myomas $(>10 \mathrm{~cm})$ were compared with myomas $<10 \mathrm{~cm}$ operated by a single surgeon ${ }^{[28]}$. While the largest myoma was $20 \mathrm{~cm}$ in diameter, operation time was the only significant difference between the two groups $(263.4 \pm 83.7 \mathrm{~min} v s$. $219.1 \pm 75.7 \mathrm{~min}, P=0.02)$. Another comparison study between myoma size $(\geq 9 \mathrm{~cm} v s .<9 \mathrm{~cm})$ showed significant increase in operation time (130 min vs. $92 \mathrm{~min})$ and estimated blood loss (100 $\mathrm{mL} v s .25 \mathrm{~mL})$, while no major adverse outcomes were reported in either group ${ }^{[29]}$. Jansen et al. ${ }^{[30]}$ retrospectively studied surgical approaches (abdominal, laparoscopic, or robotic) of myomectomies for extreme myoma burden (total specimen weight $436 \mathrm{~g}$ or $\leq 7$ myomas). While the perioperative outcomes (estimated blood loss, blood transfusion, and complications) were similar in all modalities, mean operating time was the longest in robotic surgery (239 $\mathrm{min}$ ) and mean hospital stay in abdominal surgery (2.2 days). Based on the analyses, the likelihood of complications increases in parallel with the myoma weight and number. The authors suggested preferring abdominal or laparoscopic approach in cases with extreme myoma weight and abdominal in cases of large number of myomas. On the other hand, Kim et al. ${ }^{[31]}$ compared 30 robotic vs. 13 open surgeries for the removal of $\geq 10$ myomas. Operating times were longer in the robotic approach (360 min vs. $180 \mathrm{~min}$ ), while length of hospital stay was shorter (2.5 days vs. 3.5 days). Because there were no conversions to laparotomy or any major complication, the authors suggested robotic approach to be an alternative to open surgery in cases with more than 10 myomas. Lee et al. ${ }^{[32]}$ recommended multiport robotic myomectomy with supraumbilical incisions in myomas larger than the umbilical level not only to ensure better cosmetic effect, but also to eliminate instrument and trocar collisions in single-port systems in a limited intrapelvic space. 


\section{FERTILITY AND OBSTETRICAL OUTCOMES AND RECURRENCE}

Robotic surgery is a suitable myomectomy approach for infertile patients. In a retrospective study, more than half of the patients became pregnant with a $70 \%$ caesarean section rate without a report of uterine rupture $^{[33]}$. Uterine rupture was also not reported in a comparison study, in which long-term pregnancy and miscarriage rates did not significantly differ after robotic assisted, laparoscopic, or abdominal myomectomy ${ }^{[34]}$. In a study where deep intramural myomas were enucleated, the pregnancy rate reached $75 \%{ }^{[35]}$. The same pregnancy rate $(70 \%)$ after robotic myomectomy was published in a Canadian cohort with $84 \%$ successful delivery or ongoing pregnancy at the time of data collection ${ }^{[36]}$. The risk of recurrence was $167 \%$ higher in laparoscopic myomectomy than in open surgery. The authors hypothesized that it is likely because of the extraction of small leiomyomas, which is less exhausting in manual removal than in the laparoscopic approach. The growth of residual myoma masses then results in newly diagnosed fibroids, which are considered recurrences ${ }^{[37]}$. Considering the better flexibility of robotic instruments, enucleation of small myomas should be more accessible, leading to lower recurrence. Another reason for higher recurrence was found to be associated with the preoperative use of GnRH agonists therapy to decrease the size of myomas $^{[38]}$.

\section{SURGICAL TECHNIQUES}

Safely extracting large and numerous myomas is often a challenge in minimal invasive surgery even for experienced surgeons. Moawad et al. ${ }^{[39]}$ presented a reproducible technique enabling fast and safe tissue containment and extraction. It consists of stringing numerous fibroids together with a barbed suture, containment using a Endocatch bag, extraction through the extended umbilical incision using the Alexis Containment and Extraction System, and finally the so-called paper roll technique for specimen extraction. Suprapubic incision is a similar technique, which serves for initial abdomen insufflation, later assistant's easy access for retraction or needle entry, and finally large tissue extraction ${ }^{[40]}$. Contained manual morcellation is in comparison with electric power morcellation associated with shorter operation time but similar postoperative opioid pain relief treatment and length of hospital stay ${ }^{[41]}$. Additionally, with the cessation of power morcellation, wound complications with the necessary mini-laparotomy for tissue extraction has not increased ${ }^{[42]}$.

Authors from South Korea proposed a new surgical technique called "locking suture on myoma (LSOM)" which replaces the tenaculum forceps, thus reducing the use of one instrument and lowering the total cost of surgery ${ }^{[43]}$. In this technique, a locking V-Loc suture is applied on the myoma after its exposure and traction can be easily performed by grasping the thread. Further locking sutures are applied as the dissection advances between the myoma and myometrium. The retrieved myomas are easily collected and extracted by grasping the threads. LSOM was also shown to be more feasible for larger, heavier, and a greater number of myomas than using the robotic tenaculum forceps, emphasizing its use especially in single-site surgery.

A very interesting technique of submucosal FIGO 2 classified myoma without endometrial injury was presented in a case study ${ }^{[4]]}$. The authors recommended several steps to prevent penetration of to the uterine cavity. Proper preoperative and intraoperative imaging is crucial for planning the surgery and determining the correct site of myometrial incision. This is followed by cold cut careful preparation of the plane between the myoma and endometrium. Infusion of indigo carmine to dilatate the uterine cavity aids in delineating the endometrial cavity during dissection.

Blood loss can be lowered without compromising surgical morbidity by the vascular control technique ${ }^{[45]}$. This method uses vascular (bulldog) clamps to temporarily occlude the uterine arteries during the myomectomy. The maximal limit of occlusion time was set at $60 \mathrm{~min}$ with $5 \mathrm{~min}$ 
reperfusion intervals every $20 \mathrm{~min}$.

One of the technical disadvantages of the robotic system is the lack of haptic feedback of the instruments. In myomectomy especially, the absence of tactile feedback can lead to longer operation time due to the necessary identification of intramural myomas with ultrasound and less accurate and destructive myometrial incisions above the myoma ${ }^{[13]}$. Giannini et al. ${ }^{[46]}$ presented a device (wearable fabric yielding display) that can reproduce the stiffness of myomas ex vivo. When integrated into commercially available robotic systems, this device could lead to a better intraoperative identification of myomas with more precise surgery.

\section{LEARNING CURVE AND ECONOMICS}

Acquisition of a new robotic system and its maintenance cost are considered as the biggest drawback of faster expansion of robotic surgery worldwide. Despite the obligatory cost of purchasing the robotic system with its disposable instruments, implementation of correct strategies can reduce the costs of robotic surgery while maximizing its benefits. The most influential modifying factors which lead to the cost-effectiveness of robotic assisted surgeries are intraoperative and postoperative complications, length of surgery, and length of hospital stay, which are all related to surgeons' experience ${ }^{[47]}$.

Even though there are no recent data on cost comparison of robotic $v s$. standard laparoscopic myomectomy, information could be related from benign hysterectomy surgeries. Interestingly, after adjusting patient-level covariates such as uterine weight, age, BMI, and previous abdominal or pelvic surgery, the cost of robotic surgery $v s$. laparoscopy was not significantly different in two separate hospitals. It is important to point out that the surgeries were performed by experienced surgeons past their learning curve ${ }^{[48]}$. Similar results were presented in a randomized trial ${ }^{[49]}$, where comparable cost could be attained between the two modalities if a robot is already a pre-existing investment.

Data from single-center experience with robotic single-site myomectomy show a very rapid learning curve $^{[50]}$. After 10 cases, port placement time and docking time significantly reduced, in addition to a higher number of retrieved myomas and lower hemoglobin decrease after surgery. When comparing with conventional laparoscopy, docking time needs to be assessed separately as it is an element that does not exist in laparoscopy. When looking at cases of robotic hysterectomies, console time has the most rapid learning curve followed by docking time. Even in the case of a well-experienced laparoscopic surgeon transferring to robotic surgery, suturing requires the greatest number of attempts to achieve stability ${ }^{[51]}$.

Given that suturing is a major part of myomectomy, the surgeon's experience is a crucial variable in operation time resulting in cost effectiveness, as stated in previous studies.

Many resident and young surgeons struggle to keep up with the rapid advances in surgical techniques, mainly due to a lack of time spent in the operating room. Surgical simulators have an important role in helping to master these techniques outside the operation theatre. While computer simulators are often expensive and not realistic enough, live simulations are often very basic and life-like models cannot reproduce complex cases. Towner et al. ${ }^{[52]}$ constructed a model of myomatosus uterus with artificial blood perfusion and secured it in a training box. In the post-simulation survey, residents stated higher confidence and comfort performing minimal invasive myomectomy which could have a positive impact on the learning curve in real-life surgeries. 


\section{CONCLUSION}

The use of robotic systems enabled the implication of the advantages of mini-invasive surgery even in more complex cases. As the conclusions of studies are still not consistent, robotic myomectomy tends to show superiority in many factors over laparoscopy and open surgery. Operating time and higher cost seem to be the major drawback, but with the application of the presented surgical techniques and steep learning curve these could be rapidly minimized. Robotic single-site surgery seems to be a further step to reduce morbidity and pain and enhance cosmetic outcomes of minimal invasive techniques. This latest surgical access seems to be a feasible and safe procedure for myomectomy, which could replace conventional laparoscopy and robotic multiport surgeries even in complicated cases.

\section{DECLARATIONS}

\section{Authors' contributions}

Made substantial contributions to concept and design of the article: Kiss I, Svobodova P, Karasek L, Svoboda B

Drafted the article: Kiss I, Svobodova P

Critical revision of the article: Svobodova P, Karasek L, Svoboda B

\section{Availability of data and materials}

Not applicable.

\section{Financial support and sponsorship}

None.

\section{Conflicts of interest}

All authors declared that there are no conflicts of interest.

\section{Ethical approval and consent to participate}

Not applicable.

\section{Consent for publication}

Not applicable.

\section{Copyright}

(c) The Author(s) 2021.

\section{REFERENCES}

1. Stewart EA, Cookson CL, Gandolfo RA, Schulze-Rath R. Epidemiology of uterine fibroids: a systematic review. BJOG 2017;124:1501-12. DOI PubMed

2. Bulun SE. Uterine fibroids. N Engl J Med 2013;369:1344-55. DOI PubMed

3. Parker WH. Etiology, symptomatology, and diagnosis of uterine myomas. Fertil Steril 2007;87:725-36. DOI PubMed

4. Vlahos NF, Theodoridis TD, Partsinevelos GA. Myomas and adenomyosis: impact on reproductive outcome. Biomed Res Int 2017;2017:5926470. DOI PubMed PMC

5. Munro MG, Critchley HOD, Fraser IS; FIGO Menstrual Disorders Committee. The two FIGO systems for normal and abnormal uterine bleeding symptoms and classification of causes of abnormal uterine bleeding in the reproductive years: 2018 revisions. Int $J$ Gynaecol Obstet 2018;143:393-408. DOI PubMed

6. Donnez J, Dolmans MM. Uterine fibroid management: from the present to the future. Hum Reprod Update 2016;22:665-86. DOI PubMed PMC

7. Donnez J, Arriagada P, Donnez O, Dolmans MM. Emerging treatment options for uterine fibroids. Expert Opin Emerg Drugs 2018;23:17-23. DOI PubMed

8. Wang T, Tang H, Xie Z, Deng S. Robotic-assisted vs. laparoscopic and abdominal myomectomy for treatment of uterine fibroids: a meta-analysis. Minim Invasive Ther Allied Technol 2018;27:249-64. DOI PubMed

9. Ranes M, Carlan SJ, Vaught J, Greves CE. Robot-assisted laparoscopic myomectomy versus abdominal myomectomy a retrospective 
comparison of short-term surgical outcomes. J Reprod Med 2016;61:416-20. PubMed

10. Iavazzo C, Mamais I, Gkegkes ID. Robotic assisted vs. laparoscopic and/or open myomectomy: systematic review and meta-analysis of the clinical evidence. Arch Gynecol Obstet 2016;294:5-17. DOI PubMed

11. Cezar C, Becker S, di Spiezio Sardo A, et al. Laparoscopy or laparotomy as the way of entrance in myoma enucleation. Arch Gynecol Obstet 2017;296:709-20. DOI PubMed

12. Pundir J, Pundir V, Walavalkar R, Omanwa K, Lancaster G, Kayani S. Robotic-assisted laparoscopic vs. abdominal and laparoscopic myomectomy: systematic review and meta-analysis. J Minim Invasive Gynecol 2013;20:335-45. DOI PubMed

13. Gingold JA, Gueye NA, Falcone T. Minimally invasive approaches to myoma management. J Minim Invasive Gynecol 2018;25:23750. DOI PubMed

14. Takmaz O, Ozbasli E, Gundogan S, et al. Symptoms and health quality after laparoscopic and robotic myomectomy. JSLS 2018;22:e2018. DOI PubMed PMC

15. Choi SH, Hong S, Kim M, et al. Robotic-assisted laparoscopic myomectomy: the feasibility in single-site system. Obstet Gynecol Sci 2019;62:56-64. DOI PubMed PMC

16. Moawad GN, Tyan P, Paek J, et al. Comparison between single-site and multiport robot-assisted myomectomy. J Robot Surg 2019;13:757-64. DOI PubMed

17. Giovannopoulou E, Prodromidou A, Blontzos N, Iavazzo C. The emerging role of robotic single-site approach for myomectomy: a systematic review of the literature. Surg Innov 2021. DOI PubMed

18. Won S, Lee N, Kim M, et al. Comparison of operative time between robotic and laparoscopic myomectomy for removal of numerous myomas. Int J Med Robot 2020;16:1-5. DOI PubMed

19. Park KM, Kang S, Kim C, et al. Variables that prolong total operative time for robotic-assisted laparoscopic myomectomy: a 10-year tertiary hospital study in Korea. Eur J Obstet Gynecol Reprod Biol 2021;262:62-7. DOI PubMed

20. Lee SR, Kim JH, Kim S, Kim SH, Chae HD. The number of myomas is the most important risk factor for blood loss and total operation time in robotic myomectomy: analysis of 242 cases. J Clin Med 2021;10:2930. DOI PubMed PMC

21. Movilla P, Orlando M, Wang J, Opoku-Anane J. Predictors of prolonged operative time for robotic-assisted laparoscopic myomectomy: development of a preoperative calculator for total operative time. J Minim Invasive Gynecol 2020;27:646-54. DOI PubMed

22. Yuk JS, Kim YA, Lee JH. Hybrid robotic single-site myomectomy using the GelPoint platform. J Laparoendosc Adv Surg Tech A 2019;29:1475-80. DOI PubMed

23. Won S, Lee N, Kim M, et al. Robotic single-site myomectomy: a hybrid technique reducing operative time and blood loss. Int J Med Robot 2020;16:e2061. DOI PubMed

24. Yoo HK, Cho A, Cho EH, et al. Robotic single-site surgery in benign gynecologic diseases: experiences and learning curve based on 626 robotic cases at a single institute. J Obstet Gynaecol Res 2020;46:1885-92. DOI PubMed

25. Takmaz Ö, Gündoğan S, Özbaşlı E, et al. Laparoscopic assisted robotic myomectomy of a huge myoma; Does robotic surgery change the borders in minimally invasive gynecology? J Turk Ger Gynecol Assoc 2019;20:211-2. DOI PubMed PMC

26. Jeong HG, Lee MJ, Lee JR, Jee BC, Kim SK. The largest uterine leiomyoma removed by robotic-assisted laparoscopy in the late reproductive age: a case report. J Menopausal Med 2021;27:37-41. DOI PubMed PMC

27. Lee SR, Lee ES, Lee YJ, et al. Robot-assisted laparoscopic myomectomy versus abdominal myomectomy for large myomas sized over $10 \mathrm{~cm}$ or weighing 250 g. Yonsei Med J 2020;61:1054-9. DOI PubMed PMC

28. Lee CY, Chen IH, Torng PL. Robotic myomectomy for large uterine myomas. Taiwan J Obstet Gynecol 2018;57:796-800. DOI PubMed

29. Gunnala V, Setton R, Pereira N, Huang JQ. Robot-assisted myomectomy for large uterine myomas: a single center experience. Minim Invasive Surg 2016;2016:4905292. DOI PubMed PMC

30. Jansen LJ, Clark NV, Dmello M, Gu X, Einarsson JI, Cohen SL. Perioperative outcomes of myomectomy for extreme myoma burden: comparison of surgical approaches. J Minim Invasive Gynecol 2019;26:1095-103. DOI PubMed

31. Kim H, Shim S, Hwang Y, et al. Is robot-assisted laparoscopic myomectomy limited in multiple myomas? Obstet Gynecol Sci 2018;61:135-41. DOI PubMed PMC

32. Lee SR, Kim JH, Lee YJ, et al. Single-incision versus multiport robotic myomectomy: a propensity score matched analysis of surgical outcomes and surgical tips. J Clin Med 2021;10:3957. DOI PubMed PMC

33. Huberlant S, Lenot J, Neron M, et al. Fertility and obstetrical outcomes after robot-assisted laparoscopic myomectomy. Int J Med Robot 2020;16:e2059. DOI PubMed

34. Flyckt R, Soto E, Nutter B, Falcone T. Comparison of long-term fertility and bleeding outcomes after robotic-assisted, laparoscopic, and abdominal myomectomy. Obstet Gynecol Int 2016;2016:2789201. DOI PubMed PMC

35. Kang SY, Jeung IC, Chung YJ, et al. Robot-assisted laparoscopic myomectomy for deep intramural myomas. Int J Med Robot 2017;13:e1742. DOI PubMed

36. Goldberg HR, McCaffrey C, Amjad H, Kives S. Fertility and pregnancy outcomes after robotic-assisted laparoscopic myomectomy in a Canadian cohort. J Minim Invasive Gynecol 2021. DOI PubMed

37. Kotani Y, Tobiume T, Fujishima R, et al. Recurrence of uterine myoma after myomectomy: open myomectomy versus laparoscopic myomectomy. J Obstet Gynaecol Res 2018;44:298-302. DOI PubMed PMC

38. Sangha R, Katukuri V, Palmer M, Khangura RK. Recurrence after robotic myomectomy: is it associated with use of GnRH agonist? $J$ Robot Surg 2016;10:245-9. DOI PubMed

39. Moawad GN, Tyan P, Awad C. Technique for tissue containment and extraction in the complex minimally invasive myomectomy 
setting. J Minim Invasive Gynecol 2019;26:809-10. DOI PubMed

40. Gueye NA, Goodman LR, Falcone T. Versatility of the suprapubic port in robotic assisted laparoscopic myomectomy. Fertil Steril 2017;108:e1. DOI PubMed

41. Sanderson DJ, Sanderson R, Cleason D, Seaman C, Ghomi A. Manual morcellation compared to power morcellation during robotic myomectomy. J Robot Surg 2019;13:209-14. DOI PubMed

42. Dubin AK, Wei J, Sullivan S, Udaltsova N, Zaritsky E, Yamamoto MP. Minilaparotomy versus laparoscopic myomectomy after cessation of power morcellation: rate of wound complications. J Minim Invasive Gynecol 2017;24:946-53. DOI PubMed

43. Lee SR, Lee ES, Eum HL, et al. New surgical technique for robotic myomectomy: continuous locking suture on myoma (LSOM) technique. J Clin Med 2021;10:654. DOI PubMed PMC

44. Hijazi A, Chung YJ, Kang HJ, Song JY, Cho HH, Kim MR. Robot-assisted laparoscopic myomectomy for FIGO type II sub-mucosal leiomyoma without endometrial injury for a patient with history of miscarriage. J Turk Ger Gynecol Assoc 2021;22:80-2. DOI PubMed PMC

45. Song T, Han YG, Sung JH. Comparison between the vascular control technique and conventional technique for reducing operative blood loss during robot-assisted myomectomy. Int J Med Robot 2019;15:e2038. DOI PubMed

46. Giannini A, Bianchi M, Doria D, et al. Wearable haptic interfaces for applications in gynecologic robotic surgery: a proof of concept in robotic myomectomy. J Robot Surg 2019;13:585-8. DOI PubMed

47. Wu CZ, Klebanoff JS, Tyan P, Moawad GN. Review of strategies and factors to maximize cost-effectiveness of robotic hysterectomies and myomectomies in benign gynecological disease. J Robot Surg 2019;13:635-42. DOI PubMed

48. Winter ML, Leu SY, Lagrew DC Jr, Bustillo G. Cost comparison of robotic-assisted laparoscopic hysterectomy versus standard laparoscopic hysterectomy. J Robot Surg ;9:269-75. DOI PubMed PMC

49. Lönnerfors C, Reynisson P, Persson J. A randomized trial comparing vaginal and laparoscopic hysterectomy vs. robot-assisted hysterectomy. J Minim Invasive Gynecol 2015;22:78-86. DOI PubMed

50. Kim M, Kim MK, Kim ML, Jung YW, Yun BS, Seong SJ. Robotic single-site myomectomy: a single-center experience of 101 consecutive cases. Int J Med Robot 2019;15:e1959. DOI PubMed

51. Tang FH, Tsai EM. Learning curve analysis of different stages of robotic-assisted laparoscopic hysterectomy. BioMed Res Int 2017;2017:1827913. DOI PubMed PMC

52. Towner MN, Lozada-Capriles Y, LaLonde A, et al. Creation and piloting of a model for simulating a minimally invasive myomectomy. Cureus 2019;11:e4223. DOI PubMed PMC 